\title{
DETERMINAÇÃO DE PESTICIDAS EM FEIJÃO POR DISPERSÃO DA MATRIZ EM FASE SÓLIDA (DMFS)
}

WANEIDE GOMES LOPES * HAROLDO SILVEIRA DÓREA *

Desenvolveu-se metodologia analítica por dispersão da matriz em fase sólida (DMFS) para analisar Diazinon, Propanil, Malation, Cipermetrina e Deltametrina em amostras de feijão, testando-se o método em amostras comerciais. Amostras de feijão foram trituradas em moinho de facas e uma subamostra de $5 \mathrm{~g}$ foi homogeneizada com $1 \mathrm{~g}$ de alumina neutra. O conteúdo da mistura foi transferido para coluna DMFS e acetato de etila foi usado para eluição. O extrato final foi analisado em cromatógrafo a gás/espectrômetro de massa (CG/EM). Amostras fortificadas entre 0,5 e $4,0 \mathrm{mg} \cdot \mathrm{kg}^{-1}$ apresentaram valores de recuperação $(n=3)$ entre $72,7 \%$ e $102,1 \%$ e média da estimativa do desvio-padrão entre $29,4 \%$ e $44,7 \%$. Os limites de detecção (LD) variaram entre 0,002 a 0,2 mg. $\mathrm{kg}^{-1}$ para os pesticidas estudados, portanto abaixo dos limites máximos de resíduos (LMR) permitidos pelo Codex Alimentarius. Na análise das amostras comerciais de feijão foram encontrados os pesticidas Diazinon, Propanil e Deltametrina em concentrações acima LMR. O método mostrou-se apropriado para pequenas quantidades de amostra, rápido e simples, com poucas etapas na preparação de amostra e pouco consumo de solvente orgânico.

PALAVRAS-CHAVE: FEIJÃO; DISPERSÃO DA MATRIZ EM FASE SÓLIDA; DIAZINON; PROPANIL; MALATION, CIPERMETRINA; DELTAMETRINA; CROMATOGRAFIA A GÁS; ESPECTRÔMETRO DE MASSA.

* Graduando em Engenharia Química, Universidade Federal de Sergipe (UFS), São Cristóvão-SE.

** Professor, Doutor em Química pelo Instituto de Química de São Carlos (IQSC), Universidade de São Paulo (USP); Departamento de Química, UFS, São Cristóvão-SE (e-mail: haroldo@ufs.br). 


\section{INTRODUÇÃO}

Grandes quantidades de pesticidas são usadas na agricultura para eliminar pragas que oferecem perigo às plantações, proporcionando benefício inquestionável na produção de gêneros alimentícios. Entretanto, resíduos de pesticidas permanecem nos alimentos, no solo, nas águas subterrâneas e nas águas de descarte após a aplicação desses compostos químicos. Resíduos de pesticidas constituem risco para a saúde humana em razão da toxicidade dos produtos. Para proteger a saúde do consumidor, muitos países têm restringido o uso dos pesticidas e estabelecido leis para controlar os níveis dos seus resíduos.

Muitos métodos que trabalham com a determinação de resíduos de pesticidas em alimentos, como o de LUKE et al. (1975), envolvem várias etapas de extração, purificação e concentração, que consomem tempo e solventes orgânicos.

A complexidade das amostras a serem analisadas e a exigência em determinar níveis cada vez menores de concentração têm demandado o surgimento de novas técnicas de extração, novos equipamentos e novos métodos para análise de resíduos de pesticidas. A etapa de preparação da amostra tem sido objeto de estudo por consumir maior tempo na análise. Técnicas como extração em fase sólida (TOLOSA et al.,1996; DÓREA et al., 1996), extração com fluido supercrítico (LANÇAS et al., 1997), extração com água subcrítica (Subcritical Water Extraction-SWE), extração de solvente acelerada (Accelerated Solvent Extraction-ASE), solvente pressurizado (Pressurized Solvent Extraction - PSE), ultra-som, microextração líquido-líquido (Micro Liquid-Liquid Extraction - $\mu$-LLE), micro-extração em fase sólida (Solid-Phase Microextraction-SPME), extração por microondas (Microwave Assisted Extraction $-M A E)$ e a técnica de extração imunoquímica estão sendo desenvolvidas para essa finalidade (LANÇAS, 2003; LLE e BARTLE, 1998).

A Dispersão da Matriz em Fase Sólida (DMFS) ou Matrix Solid Phase Dispersion (MSPD), desenvolvida para amostras sólidas e semi-sólidas (BARKER, 1989) tem como principais características o uso de pequenas quantidades de amostras, pouco consumo de solvente orgânico, a realização da extração numa única etapa com o clean up, baixo custo e rapidez na extração (DÓREA e LANÇAS, 1999). Essa técnica foi introduzida no Brasil para analisar pesticidas em frutas (DÓREA, 1999) e outros trabalhos têm sido desenvolvidos para análise de pesticidas em grãos (LIMA SOBRINHO, 2001), peixe (LOTT et al, 1993), leite (SCHENCK et al., 1996), vegetais (VIANA et al., 1996) e em plantas medicinais (ZUIN et al., 2003).

Desenvolveu-se, neste trabalho, método por dispersão da matriz em fase sólida para analisar os pesticidas organofosforados Diazinon e Malation, os Piretróides sintéticos Cipermetrina e Deltametrina, bem como Propanil (anilida) em amostras de feijão.

\section{MATERIAL E MÉTODOS}

\subsection{REAGENTES E SOLUÇÕES}

Foram utilizados acetato de etila (Merck), alumina neutra (Fluka), sílica gel (Merck) e adquiridos padrões de Diazinon (AccuStandard), Propanil (Riedel), Malation (Radian), Cipermetrina (AccuStandard) e Deltametrina (AccuStandard).

As soluções-padrão foram preparadas em acetato de etila com concentrações diferenciadas, segundo a sensibilidade do composto no detector de espectrometria de massas. 


\subsection{PROCESSO POR DISPERSÃO DA MATRIZ EM FASE SÓLIDA (DMFS)}

O processo para extração de pesticidas organofosforados e Piretróides em feijão pela DMFS consiste em adicionar pequena quantidade do dispersante alumina neutra $\left(\mathrm{Al}_{2} \mathrm{O}_{3}\right)$ à amostra de feijão finamente triturada, homogeneizá-la por alguns minutos e transferi-la para a coluna. Pode-se usar cartucho DMFS comercial ou prepará-la no laboratório momentos antes da extração. Coloca-se a mistura amostra-dispersante em seringa de injeção de polietileno comum (vendida nas farmácias), tendo lã de vidro como base de sustentação para o sólido que constituirá a coluna DMFS. Sob a mistura sólida pode ser adicionado sulfato de sódio anidro $\left(\mathrm{Na}_{2} \mathrm{SO}_{4}\right)$ para reter a água presente na amostra, caso seja necessário. A eluição pode ser efetuada com bombas de vácuo ou por fluxo gravitacional (recomendando-se usar vácuo nos cartuchos DMFS comerciais). A escolha do solvente de eluição deve recair nos parâmetros utilizados para extração em fase sólida, isto é, no melhor compromisso entre interação eluente-analito e eluente-interferente. Solvente para retirar interferentes também pode ser usado antes da eluição dos pesticidas. Esse é o processo DMFS sem co-coluna in line, usada normalmente para melhorar o clean up do extrato final. A co-coluna pode ser outra fase sólida, colocada sob a mistura amostra-adsorvente na coluna DMFS. Também pode ser usada outra coluna de polietileno contendo a fase sólida de interesse colocada abaixo da coluna DMFS (duas colunas acopladas verticalmente). O volume de solvente orgânico usado como eluente deve ser testado, visando reduzi-lo ao mínimo necessário para extrair todos os analitos (pesticidas) e retirar o mínimo dos compostos endógenos da amostra (interferentes). A concentração da amostra é a etapa final do processo (evaporação em banho de água a vácuo) para a análise cromatográfica.

\subsection{PREPARO DA AMOSTRA POR DMFS}

O procedimento para a etapa de preparo da amostra por dispersão da matriz em fase sólida, desenvolvido nesse trabalho, é apresentado na Figura 1. Amostras de feijão não-contaminadas foram utilizadas para o desenvolvimento da metodologia. Para o teste final foram coletadas amostras comerciais. Após recebimento das amostras, as mesmas foram moídas em moinho de facas (Willye TE650, Tecnal) e acondicionadas em bolsas plásticas, vedadas, identificadas e conservadas em freezer. No momento da extração as amostras foram retiradas e, após atingirem a temperatura ambiente, pesadas. O extrato final foi preservado em freezer até o momento da análise em cromatógrafo a gás/ espectrômetro de massa (CG/EM).

\subsection{CONDIÇÕES CROMATOGRÁFICAS}

As análises foram realizadas em cromatógrafo a gás, acoplado a espectrômetro de massas Shimadzu, modelo QP5050A, equipado com injetor split/splitless $\left(250^{\circ} \mathrm{C}\right)$, coluna DB-5 $(30 \mathrm{~m} \mathrm{x}$ $0,25 \mathrm{~mm} \times 0,25 \mu \mathrm{m}$ ), com programação de temperatura iniciando a $140^{\circ} \mathrm{C}$ (1 $\left.\mathrm{min}\right)$, taxa de crescimento de $15^{\circ} \mathrm{C} / \mathrm{min}$ até $300^{\circ} \mathrm{C}(6 \mathrm{~min})$. A temperatura da interface entre $\mathrm{CG} / \mathrm{MS}$ foi de $280^{\circ} \mathrm{C}$. Hélio foi usado como gás de arraste (101 kPa), razão de split 1:10, sendo $1 \mu \mathrm{L}$ de solução-padrão ou extrato da amostra injetado no cromatógrafo. O espectrômetro de massas foi operado no modo SIM (single ion monitoring), com fonte de íons de impacto de elétrons a $70 \mathrm{eV}$. Os fragmentos selecionados foram $127,161,179$ e $181 \mathrm{~m} / \mathrm{z}$ para os pesticidas estudados. 


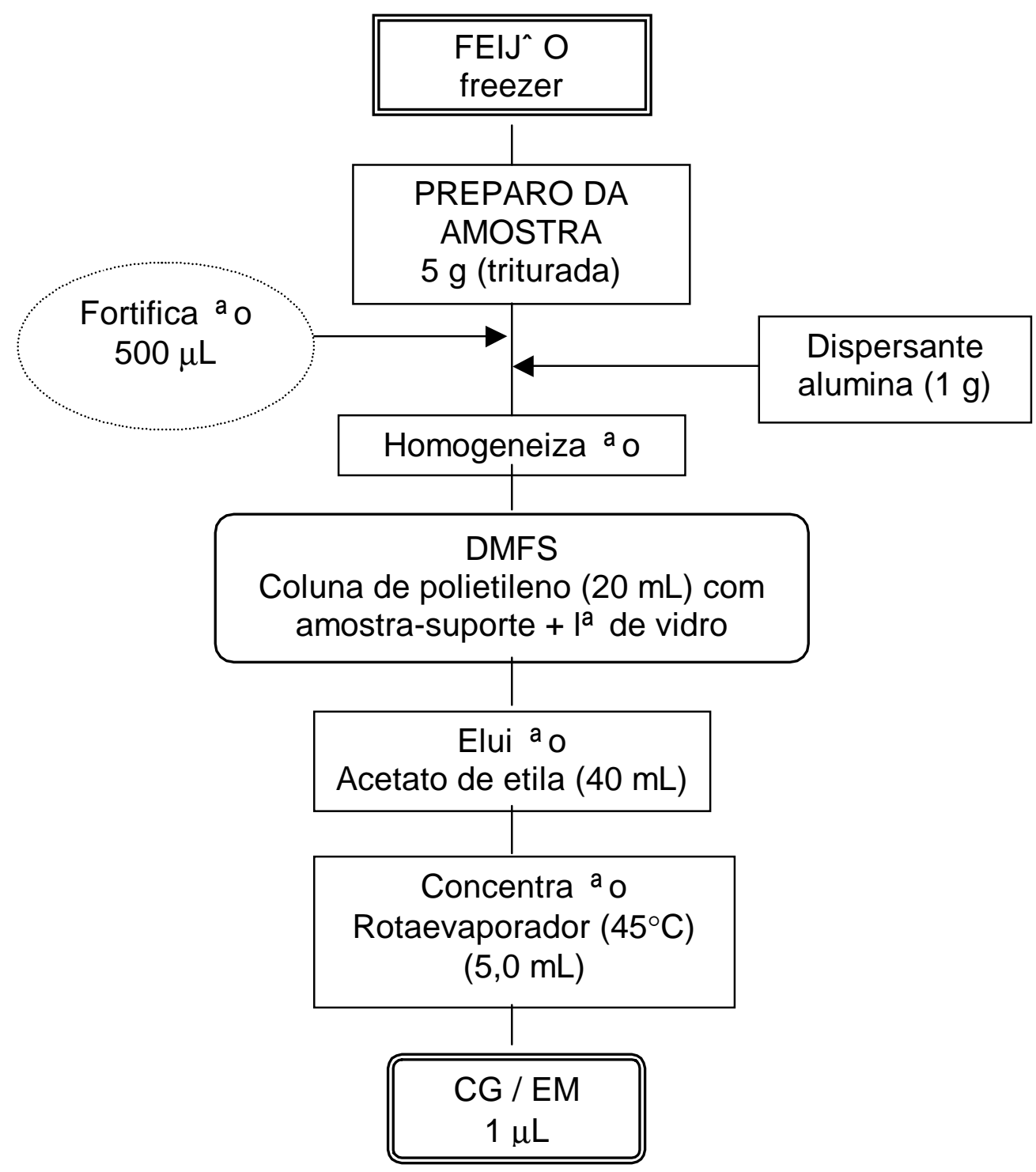

\section{RESULTADOS E DISCUSSÃO}

A escolha dos pesticidas para esse trabalho baseou-se em levantamento efetuado por LIMA SOBRINHO (2001) no estado de Sergipe para a cultura de feijão e de outros grãos. Em seguida, foram desenvolvidas as condições cromatográficas (CG/EM), injetando-se a solução-padrão inicialmente no modo Scan. Efetuou-se a seleção dos íons para cada composto e estabeleceu-se o método no modo SIM para os resultados quantitativos (Tabela 1 ).

A quantificação foi efetuada pelo método padrão externo, com a curva analítica obtida pelo método dos mínimos quadrados para o intervalo de 0,3 a $8,0 \mu \mathrm{g} / \mathrm{mL}$ para os pesticidas (Tabela 1 ). 
TABELA 1 - TEMPO DE RETENÇÃO, MASSA MOLECULAR, ÍON SELECIONADO PARA O DETECTOR DE MASSAS, EQUAÇÃO DA RETA E COEFICIENTE DE CORRELAÇÃO DAS CURVAS ANALÍTICAS DOS PESTICIDAS ESTUDADOS

\begin{tabular}{lcccccc}
\hline Pesticida & $\begin{array}{c}\mathrm{tr} \\
(\mathrm{min})\end{array}$ & $\begin{array}{c}\mathrm{M} \\
\left(\mathrm{g} \cdot \mathrm{mol}^{-1}\right)\end{array}$ & $\begin{array}{c}\text { SIM } \\
(\mathrm{m} / \mathrm{z})\end{array}$ & Equa ${ }^{\circ}$ o da Reta & $r$ \\
\hline Diazinon & 6,9 & 304 & 179 & $2,4.10^{4} \times$ & $4,9.10^{3}$ & 0,9966 \\
Propanil & 7,5 & 217 & 161 & $5,2.10^{4} \times-2,3.10^{4}$ & 0,9999 \\
Malation & 8,1 & 330 & 127 & $7,4.10^{4} \times$ & $8,6.10^{3}$ & 0,9973 \\
Cipermetrina-Cis & 13,5 & 415 & 181 & $7,9.10^{3} \times$ & $7,3.10^{3}$ & 0,9996 \\
Cipermetrina-Trans & 13,7 & 415 & 181 & $7,0.10^{3} \times$ & $6,7.10^{3}$ & 0,9998 \\
Deltametrina & 15,6 & 503 & 181 & $7,1.10^{3} \times$ & $5,9.10^{3}$ & 0,9911
\end{tabular}

$\mathrm{Tr}=$ Tempo de retenção.

$\mathrm{M}=$ Massa molecular.

$\mathrm{SIM}=$ Íon selecionado para o detector de massas.

Foram realizados testes com os dispersantes (adsorventes) sílica e alumina neutra em várias proporções. Também foram feitos testes com e sem co-coluna. Melhores resultados de recuperação foram obtidos utilizando-se alumina como dispersante. A co-coluna mostrou-se desnecessária nas condições utilizadas com acetato de etila como eluente. Devido aos bons resultados obtidos em trabalhos anteriores (DÓREA e LANÇAS, 1999; LIMA SOBRINHO, 2001; TORRES et al., 1995 e 1996), o acetato de etila foi usado como eluente.

A principal modificação do método desenvolvido por dispersão da matriz em fase sólida (DMFS) para analisar pesticidas organofosforados e piretróide em feijão foi o uso do adsorvente alumina como dispersante. Nos métodos convencionais por DMFS a etapa de dispersão é realizada geralmente com octadecilsilano $\left(\mathrm{C}_{18}\right)$.

Os padrões de pesticidas foram adicionados às amostras de feijão não-contaminadas antes da etapa de homogeneização, aguardando-se intervalo para que houvesse maior contato dos pesticidas com a amostra. Devido à pequena quantidade de amostra utilizada, a adição de pequeno volume da solução-padrão contendo os pesticidas torna-se necessária para evitar perdas do padrão nas paredes do recipiente.

\subsection{RECUPERAÇÕES E DESVIOS DO MÉTODO POR DMFS}

A Tabela 2 mostra o resultado de recuperação e a estimativa do desvio-padrão relativo (DPR) dos pesticidas estudados. Amostras de feijão fortificadas entre 0,5 e $4,0 \mathrm{mg} \cdot \mathrm{kg}^{-1}$ apresentaram valores de recuperação $(n=3)$ entre $72,7 \%$ e 102,1\%, com 90,3\% de recuperação média (DPR médio de $34,6 \%$ ) para todos os pesticidas. A Cipermetrina foi analisada como dois compostos independentes, devido aos isômeros cis e trans que foram separados na coluna cromatográfica, mas a média de suas recuperações foi de $97,6 \%$ com DPR médio de $33,5 \%$. Os resultados de recuperação obtidos evidenciaram a potencialidade da técnica. 


\section{TABELA 2 - RESULTADOS DE RECUPERAÇÃO $(n=3)$ E ESTIMATIVA DO DESVIO-PADRÃO RELATIVO DOS PESTICIDAS ESTUDADOS EM AMOSTRAS DE FEIJÃO COM O MÉTODO PROPOSTO POR DMFS}

\begin{tabular}{lccc}
\hline Pesticidas & $\begin{array}{c}\text { Fortifica } \stackrel{\text { a }}{\left(\mathrm{mg}_{\mathrm{kg}}{ }^{-1}\right)} \\
\text { Diazinon }\end{array}$ & $\begin{array}{c}\text { Recupera } \mathrm{a} \text { o } \\
\text { MØdia }(\%)\end{array}$ & $\begin{array}{c}\text { DPR } \\
(\%)\end{array}$ \\
Propanil & 1,00 & 82,0 & 29,4 \\
Malation & 2,0 & 102,1 & 44,7 \\
Cipermetrina- cis & 0,5 & 89,8 & 31,9 \\
Cipermetrina-trans & 3,0 & 99,8 & 33,6 \\
Deltametrina & 3,0 & 95,4 & 33,4 \\
\hline
\end{tabular}

DPR = Desvio-padrão relativo.

\subsection{LIMITES DE DETECÇÃO E QUANTIFICAÇÃO POR DMFS}

A sensibilidade do método foi considerada em relação aos limites de detecção e determinação, além do nível de ruído do instrumento. Preparou-se o branco e os pesticidas foram adicionados ao extrato do branco na quantidade desejada. O limite de detecção (LD) foi definido como a resposta três vezes superior à altura média do ruído da linha de base do branco. O limite de quantificação (LQ) foi definido como a resposta dez vezes superior à altura média do ruído da linha de base do branco, nas condições cromatográficas estabelecidas e considerando a quantidade de subamostra utilizada no método. Os resultados variaram entre 0,002 e $0,2 \mathrm{mg} \cdot \mathrm{kg}^{-1}$ para LD e 0,006 a $0,7 \mathrm{mg} \cdot \mathrm{kg}^{-1}$ para LQ (Tabela 3). Os Piretróides Cipermetrina e Deltametrina mostraram sensibilidade três vezes menor que os demais pesticidas.

\section{TABELA 3 - LIMITES DE DETECÇÃO E QUANTIFICAÇÃO DOS PESTICIDAS ESTUDADOS COM O MÉTODO PROPOSTO POR DMFS, COMPARADOS COM O LIMITE MÁXIMO DE RESÍDUOS (LMR) PERMITIDO PELA FAO/OMS}

\begin{tabular}{lccc}
\hline Pesticidas & $\begin{array}{c}\mathrm{LD} \\
\left(\mathrm{mg} \cdot \mathrm{kg}^{-1}\right)\end{array}$ & $\begin{array}{c}\mathrm{LQ} \\
\left(\mathrm{mg} \cdot \mathrm{kg}^{-1}\right)\end{array}$ & $\begin{array}{c}\mathrm{LMR}^{*} \\
\left(\mathrm{mg} \cdot \mathrm{kg}^{-1}\right)\end{array}$ \\
\hline Diazinon & 0,03 & 0,09 & 0,1 \\
Propanil & 0,06 & 0,2 & $-* *$ \\
Malation & 0,002 & 0,006 & 8 \\
Cipermetrina- cis & 0,15 & 0,5 & 0,5 \\
Cipermetrina-trans & 0,2 & 0,7 & 1 \\
Deltametrina & 0,2 & 0,6 & 0,6 \\
\hline
\end{tabular}

* Limite máximo de resíduos permitido pelo Codex Alimentarius (FAO/OMS, 1994).

** Esse pesticida não consta na lista do Codex. 


\subsection{COMPARAÇÃO COM OUTROS MÉTODOS POR DMFS}

Comparado com outros métodos por dispersão da matriz em fase sólida para analisar pesticidas em alimentos, o método proposto apresenta a vantagem de não utilizar a co-coluna. Tal fato se deve, provavelmente, ao tipo da matriz usada (feijão) e as interações que ocorreram entre essa, os pesticidas estudados, o dispersante alumina neutra e o eluente acetato de etila.

TORRES et al. (1996) analisaram 18 pesticidas em laranjas, dentre os quais o Diazinon, Metidation e Malation. O método consistiu em homogeneização da amostra de $0,5 \mathrm{~g}$ com $0,5 \mathrm{~g}$ de $\mathrm{C}_{18}$ e transferência para coluna, contendo $0,5 \mathrm{~g}$ de sílica, eluição com $10 \mathrm{~mL}$ de acetato de etila e análise por CGC/ECD. Diversos parâmetros foram estudados, como dispersante e fase sólida diferentes em várias proporções. $\mathrm{O}$ uso de Florisil e alumina proporcionou menores recuperações do que o emprego de sílica. Acetona, $\mathrm{N}, \mathrm{N}$-dimetil formamida, metanol, $\mathrm{n}$-hexano, éter de petróleo e éter dietil também foram testados como eluentes. Os resultados de recuperação do método proposto para o Diazinon foi de $94 \% \pm 6 \%$, para Malation de $87 \% \pm 10 \%$ e para Metidation de $85 \% \pm 2 \%$. O método foi avaliado com amostras de laranjas adquiridas no mercado, sendo o Paration metil, o Malation e o Clorpirifós os pesticidas mais encontrados.

TORRES et al. (1995) também analisaram 16 pesticidas em frutas (limão, laranja, pomelo, pêra e ameixa) e vegetais, com diferentes detectores (ECD, NPD, FPD-P, FPD-S e MSD). Usaram o mesmo método por DMFS, a exceção da coluna contendo $1,5 \mathrm{~g}$ de sílica. Diazinon, Malation e Metidation novamente foram selecionados e as recuperações em ECD variaram entre $63 \%$ e $87 \%$ para limão e $60 \%$ a $92 \%$ para as demais frutas, com apenas uma recuperação acima de $90 \%$. Esses resultados evidenciam a dificuldade em extrair totalmente tais compostos em amostras de frutas. $O$ detector ECD mostrou-se o mais sensível dentre os testados, porém o menos seletivo. O Metidation foi o composto mais encontrado nas amostras de frutas comerciais.

Utilizando a técnica DMFS, KADENCKI et al. (1992) desenvolveram um método que chamaram simplesmente de extração por coluna (atribuindo a si mesmos a primeira publicação dessa metodologia em 1985). Entretanto, a etapa de clean up é efetuada separadamente da extração DMFS. A partir de $5 \mathrm{~g}$ da amostra de frutas ou vegetais foram adicionados 8-12 $\mathrm{g}$ de Florisil, seguido de homogeneização e transferência para coluna contendo $5 \mathrm{~mm}$ de sulfato de sódio anidro. A eluição ocorreu com acetato de etila ou diclorometano-acetona (9:1), volume de $50 \mathrm{~mL}$, e velocidade de fluxo de $5 \mathrm{~mL} / \mathrm{min}$. Para a análise por CGC/ECD foi necessária a etapa de clean up com coluna de sílica gel.

Florisil como dispersante foi utilizado por LING e HUANG (1995), para analisar seis Piretróides em vegetais. O método consistiu em homogeneizar $5 \mathrm{~g}$ da amostra em $8 \mathrm{~g}$ de Florisil e transferir para coluna contendo $0,5 \mathrm{~g}$ de Florisil e $1 \mathrm{~cm}$ da coluna com sulfato de sódio anidro para retenção da água da amostra. A combinação $\mathrm{n}$-hexano-acetona $(9: 1)$ com volume de $60 \mathrm{~mL}$ foi usada como eluente. Eluição com acetato de etila foi testada entre $20 \mathrm{~mL}$ e $100 \mathrm{~mL}$, porém a extração dos Piretróides não alcançou $80 \%$. Já com a combinação hexano-acetona (volume de $40 \mathrm{~mL}$ ) mais de $90 \%$ dos piretróides foram extraídos. A recuperação da Permetrina nas cinco amostras de vegetais variou entre $95 \%$ a 101\%. Justificaram o uso de Florisil porque materiais polares como clorofilas, triglicerídios e fitosteróis, presentes em vegetais, são associados com a superfície desse adsorvente. Tal fato é atribuído à capacidade do silicato de magnésio em absorver lipídios de forma similar ao caráter solubilizante de lipídios em $\mathrm{C}_{18}$ por DMFS. Como os Piretróides hidrofóbicos ficam ligados fracamente ao adsorvente foi necessário encontrar combinação de solventes e clean up apropriados para não extrair demasiados compostos da matriz. Os estudos mostraram que a maioria das clorofilas poderiam ser retidas em Florisil ou alumina neutra, mas não em sulfato de sódio anidro ou $\mathrm{C}_{18}$. 


\subsection{APLICAÇÃO EM AMOSTRA COMERCIAL}

Os resultados para as amostras comerciais foram obtidos corrigindo-se os valores de recuperação do método proposto. Nas amostras de feijão, coletadas no mercado de Aracaju e analisadas em triplicata, foram encontrados os pesticidas Diazinon, Propanil e Deltametrina nas concentrações de 0,$64 ; 0,39$ e $1,83 \mathrm{mg} \cdot \mathrm{kg}^{-1}$, respectivamente. A Figura 1 mostra o cromatograma do íon total de uma amostra de feijão comercial com os pesticidas determinados.

\section{FIGURA 2 - CROMATOGRAMA DO ÍON TOTAL DA AMOSTRA DE FEIJÃO COMERCIAL ANALISADA POR DMFS COM A PRESENÇA DOS PESTICIDAS (1) DIAZINON, (2) PROPANIL E (3) DELTAMETRINA}

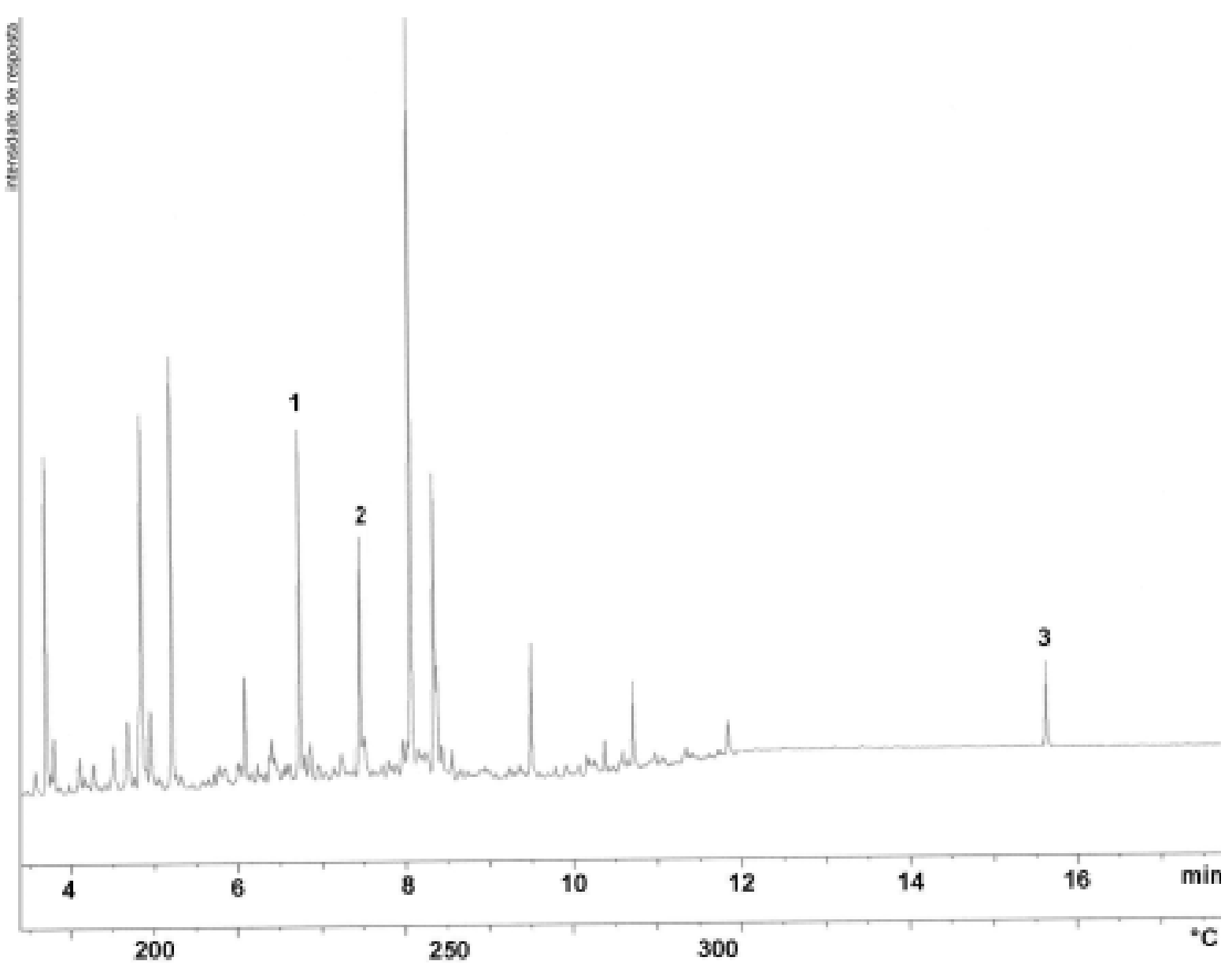

\section{CONCLUSÃO}

O método por dispersão da matriz em fase sólida (DMFS) proposto mostrou-se eficiente para analisar os pesticidas de três classes químicas diferentes (Diazinon, Propanil, Malation, Cipermetrina e Deltametrina) em amostras de feijão. Amostras fortificadas entre 0,5 e 4,0 mg. $\mathrm{kg}^{-1}$ apresentaram valores de recuperação $(n=3)$ entre $72,7 \%$ e $102,1 \%$ e estimativa do desvio-padrão entre $29,4 \%$ e $44,7 \%$. Os limites de detecção (LD) variaram entre 0,002 e $0,2 \mathrm{mg} \cdot \mathrm{kg}^{-1}$ para os pesticidas estudados, portanto abaixo dos limites máximos de resíduos exigidos pelo Codex Alimentarius. Na análise das 
amostras comerciais de feijão foram detectados os pesticidas Diazinon, Propanil e Deltametrina em concentrações acima do permitido. O método mostrou-se apropriado para pequenas quantidades de amostra, rápido e simples, com poucas etapas de preparação de amostra e pouco consumo de solvente orgânico.

\begin{abstract}
DETERMINATION OF PESTICIDES IN BEANS BY MATRIX SOLID PHASE DISPERSION (MSPD)

An analytical methodology based on matrix solid phase disperson (MSPD) was developed to analyse Diazinon, Propanil, Malathion, Cypermethrin and Deltamethrin in beans samples, by testing the method in commercial samples. Beans samples were triturated in knives mill and one sub-sample of $5 \mathrm{~g}$ was homogeneized with $1 \mathrm{~g}$ of neutral alumine. The mixture content was transferred to MSPD column and ethyl acetate was used for elution. The final extract was analysed by gas chromatograph/mass spectromethers (CG/MS). Fortified samples between 0.5 to $4 \mathrm{mg}_{\mathrm{kg}} \mathrm{k}^{-1}$ showed recuperation values $(n=3)$ between $72.7 \%$ and $102.1 \%$ and the standard deviation estimative average between $29.4 \%$ and $44.7 \%$. The detection limits varied between 0.002 to $0.2 \mathrm{mg} \cdot \mathrm{kg}^{-1}$ for the studied pesticides, though below the maximum limits of residues (MLR) permitted by Codex Alimentarius. In commercial beans samples analysis the pesticides Diazinon, Propanil, Malathion, Cypermethrin and Deltamethrin were found in concentration above the MLR. The methods was appropriated for small sample quantities, fast and simple, with few stages in sample preparation and low use of organic solvents.

KEY-WORDS: BEANS; MATRIX SOLID PHASE DISPERSION; DIAZINON; PROPANIL; MALATHION, CYPERMETHRIN, DELTAMETHRIN; GAS CHROMATOGRAPHY; MASS SPECTROMETER.
\end{abstract}

\title{
REFERÊNCIAS
}

1 BARKER, S.A.; LONG, A. R.; SHORT, C.R. Isolation of drug residues from tissues by solid phase dispersion. Journal of Chromatography, v.475, p.353-361, 1989.

2 DÓREA, H.S. Análise multirresíduo de pesticidas organofosforados e piretróides em frutas por CGC, após extração com fluido supercrítico: comparação com LLE, SPE, e MSPD. São Carlos, 1999. 214 p. Tese (Doutorado) - Instituto de Química de São Carlos, Universidade de São Paulo.

3 DÓREA, H. S, LANÇAS, F. M. Matrix Solid-Phase Dispersion Extraction of Organophosphorus and Synthetic Pyrethroid Pesticides in Cashew Nut and Passion Fruit. Journal Microcolumn Separations, v.11, n.5, p.367-375, 1999.

4 DÓREA, H.S.; TADEO, J. L.; SANCHEZ-BRUNETE, C. Determination of organophosphorus pesticides residue in fruits by gas chromatography with ITD and NPD detection. Chromatographia, v.43, n.7/8, p.380-386, 1996.

5 FAO/OMS. Food and Agriculture Organization. Codex alimentarius. $2^{\text {nd }}$ ed. Roma, 1994. v.2, 495 p.

6 KADENCZKI, L.; ARPAD, Z.; GARDI, I.; AMBRUS, A.; GYORFI, L.; REESE, G.; EBING, W. Column extraction of residues of several pesticides from fruits and vegetables: a simple multiresidue analysis method. Journal of Association Official Analytical Chemistry International, v.75, n.1, p.53-61, 1992.

7 LANÇAS, F. M.; The role of the separation sciences in the $21^{\text {th }}$ century. Journal of the Brazilian Chemical Society, Campinas, v.14, n.2, p.183-197, 2003.

8 LANÇAS, F.M.; DÓREA, H.S.; MÜLLER, R.A.; FAGUNDES, C.B. Novas aplicações de sistemas SFE "Home-Made". IV. Qualidade dos alimentos. Ciência e Tecnologia dos Alimentos, Campinas, v.17, n.4, p.432-436, 1997.

9 LEE, M.; BARTLE, K.D. Supercritical fluid extraction and supercritical fluid chromatography. In: LATIN AMERICAN CONGRESS OF CHROMATOGRAPHY AND RELATED TECHNIQUES, 7., São Pedro, SP, 1998. Short Course. São Pedro, SP: Universidade de São Paulo, Instituto de Química, 1998.

10 LIMA SOBRINHO, Ledjane. Análise multirresíduo de pesticidas no arroz por MSPD. Aracaju, 2001. $51 \mathrm{p}$. Monografia (Química Bacharelado), Departamento de Química, Universidade Federal de Sergipe.

11 LING, Y.C.; HUANG, I.P. Multi-residue matrix solid-phase dispersion method for the determination of six synthetic pyrethroids in vegetables followed by gas chromatography with electron capture detection. Journal of Chromatography A, v.695, p.75-82, 1995.

12 LOTT, H. M.; BARKER, S. A. Extraction and gas chromatographic screening of 14 chlorinated pesticides in crayfish (Procambarus clarkii) Hepatopancreas. Journal of Association Official Analytical Chemistry International, v.76, n.3, p.663-668, 1993

13 LUKE, M. A.; FROBERG, J. E.; MASUMOTO, H. T. Extraction and cleanup of organochlorine, organophosphate, organonitrogen and hydrocarbon pesticides in produce for determination by gas-liquid chromatography. Journal of 
the Association of Official Analytical Chemists, Washington, v.58, n.5, p.1020-1026, 1975.

14 SCHENCK, F. J.; CALDERON, L.; SAUDARG, D. E. Florisil solid-phase extraction cartridges for cleanup of organochlorine pesticide residues in foods. Journal of Association Official Analytical Chemistry International, v.79, n.6, p.1454-1458, 1996.

15 TOLOSA, I.; READMAN, J.W.; MEE, L.D. Comparison of the performance of solid-phase extraction techniques in recovering organophosphorus and organochlorine compounds from water. Journal of Chromatography A, v.725, p.93-106, 1996.

16 TORRES,C.M.; PICÓ, Y.; MAÑES, J. Analysis of pesticides in fruit and vegetables by matrix solid phase dispersion (MSPD) and different gas chromatography element-selective detectors. Chromatographia, v.41, n.11/12, p.685692, 1995.

17 TORRES,C.M.; PICÓ, Y.; REDONDO, M.J.; MAÑES, J. Matrix solid-phase dispersion extraction procedure for multiresidue pesticide analysis in oranges. Journal of Chromatography A, v.719, p.95-103, 1996.

18 VIANA, E.; MOLTÓ, J. C.; FONT, G. Optimization of a matrix solid phase dispersion method analysis of pesticide residues in vegetables. Journal of Chromatography A, v.754, p.437-444, 1996.

19 ZUIN, V. G.; YARIWAKE, J. H.; LANÇAS, F. M.; Analysis of pesticide residues in brazilian medicinal plants: matrix solid phase dispersion versus conventional (european pharmacopoeia) methods, Journal of the Brazilian Chemical Society, Campinas, v.14, n.2, p.304-309, 2003.

\section{AGRADECIMENTOS}

Ao CNPq pela bolsa de Iniciação Científica de Waneide G. Lopes e o apoio financeiro FINEP/CTPETRO. 